\title{
Real-Time Tool Condition Monitoring Using Wavelet Transforms and Fuzzy Techniques
}

\author{
Xiaoli Li, Shiu Kit Tso, Senior Member, IEEE, and Jun Wang, Senior Member, IEEE
}

\begin{abstract}
In this paper, wavelet transforms and fuzzy techniques are used to monitor tool breakage and wear conditions in real time according to the measured spindle and feed motor currents, respectively. First, the continuous and discrete wavelet transforms are used to decompose the spindle and feed ac servo motor current signals to extract signal features so as to detect the breakage of drills successfully. Next, the models of the relationships between the current signals and the cutting parameters are established under different tool wear states. Subsequently, fuzzy classification methods are used to detect tool wear states based on the above models. Finally, the two methods above are integrated to establish an intelligent tool condition monitoring system for drilling operations. The monitoring system can detect tool breakage and tool wear conditions using very simple current sensors. Experimental results show that the proposed system can reliably detect tool conditions in drilling operations in real time and is viable for industrial applications.
\end{abstract}

Index Terms-Current signals, fuzzy logic, tool condition monitoring, wavelet transforms.

\section{INTRODUCTION}

$\mathbf{R}$ EAL-TIME tool condition monitoring is one of the most important techniques to be developed in the automatic cutting processes as it can help to prevent damages of machine tools and workpieces. Researchers and engineers have been looking for ways to monitor tool conditions, but very few reliable monitoring systems exist nowadays in industry [1]. Direct measurement of tool wear using optical methods can only be applied when cutting tools are not in contact with the workpiece [2]. Indirect methods that rely on the relationship between tool conditions and measurable signals (such as force, acoustic emission, vibration, current, etc.) for detecting tool conditions have been extensively studied. Among the indirect methods, the method based on sensing motor current is considered as one of the major methods. Mannan et al. [3] discussed the feasibility of the motor power and current sensing for adaptive control and tool condition monitoring. Mannan et al. [4] described the use of current signals measured from spindle motor and feed motor to estimate static torque and thrust for monitoring tool conditions. The major advantage of using the measurement of motor current to monitor tool conditions in cutting processes is that fixed current

Manuscript received October 1, 1998; revised October 1, 1999 and April 1, 2000.

$\mathrm{X}$. Li is with the Institute of Precision Engineering, Harbin Institute of Technology, Harbin, 150001, China (e-mail: me150001@ cityu.edu.hk).

S. K. Tso is with the Department of Manufacturing Engineering, City University of Hong Kong, Hong Kong.

J. Wang is with the Department of Mechanical and Automation Engineering,

The Chinese University of Hong Kong, Hong Kong.

Publisher Item Identifier S 1094-6977(00)09087-8. sensors do not disturb machining processes, and the cost of the sensors is very low [5].

Signal processing is a very important step for tool condition monitoring. Recently, wavelet transforms (WT) has provided a significant new technique in signal processing, because it offers solutions in the time-frequency domain and is able to extract more information in the time domain at different frequency bands. There have been many research activities in the applications of wavelet transforms for tool condition monitoring [6]-[13]. In [6], Li discusses the use of wavelet transforms to decompose acoustic emission signals and the root mean square (RMS) values of the decomposed signals are taken as tool wear monitoring features. Gong et al. [7] proposed to use wavelet transforms to analyze cutting force signals and wavelet transform coefficients are taken as recognition parameters of flank wear states. The experimental and analytical results show that in monitoring the flank wear states during turning operations, the wavelet analysis is more sensitive, more reliable and faster than the Fourier analysis. Wu et al. [9] introduced a new method of feature extraction and assessment using a wavelet packet transform for monitoring of machining processes. Zhou et al. [10] used neural networks and wavelet techniques to propose a new tool-life criterion. Kasashima et al. [11] described an application of the discrete wavelet transform (DWT) to detecting tool failures in face milling operations. Tansel et al. [12] applied wavelet transform to compress cutting force signal. Tansel et al. [13] proposed encoding of thrust force signals of microdrilling operations with wavelet transformations for detection of severe tool damage just before complete tip breakage occurs. There has hence been much interest in the wavelet transform method for tool condition monitoring. In this paper, wavelet transforms are applied to detect the breakage of drills.

Most of the indirect approaches have been developed for fixed cutting conditions. In practical applications, however, cutting conditions are not fixed. Spindle and feed speed might change due to control strategies (e.g., reducing the feed before approaching corner points to avoid over cut). Therefore, a wear estimation strategy that operates under varying cutting conditions is needed [14].

In this paper, wavelet transforms and fuzzy technique are used to monitor tool condition with spindle motor and feed motor current signals. In Section II, the spindle and feed ac servo motor current signals are decomposed by continuous and discrete wavelet transforms, the signal features of tool breakage are extracted from the decomposed current signals and tool breakage is successfully monitored by the signal features. In Section III, regression and fuzzy classification are used to model the relationship between the measured current value and 
the tool wear states over a wide range of cutting conditions. Based on the models, tool wear condition can be estimated by the known cutting parameters and cutting current signals. In Section IV, tool breakage detection with wavelet transforms and tool wear estimation with fuzzy classification are integrated to establish an intelligent tool condition monitoring system for drilling operations, and the validity of the monitoring method is verified by means of experimental results.

\section{ToOl BREAKAgE Detection Using WAVELET TRANSFORMS}

\section{A. Wavelet Transforms}

Wavelet transformation has become well known as a useful tool for various signal processing applications [15]. Given a time-varying signal $f(t)$, a wavelet transform consist of computing coefficients that are inner products of the signal and a family of wavelets. To consider the continuous wavelet transform, the wavelet corresponding to scale $a$ and time location $b$ is [16]

$$
\psi_{a, b}=\frac{1}{\sqrt{|a|}} \psi\left(\frac{t-b}{a}\right) \quad a, b \in R, \quad a \neq 0
$$

where $a$ and $b$ are the dilation and translation parameters, respectively.

The continuous wavelet transform is then defined as follows:

$$
\mathrm{CWT}\{x(t) ; a, b\}=\int x(t) \psi_{a, b}^{*}(t) d t
$$

where "*" denotes the complex conjugation. When $a=2^{j}, b=$ $k 2^{j}, j, k \in Z$, (1) can be written as

$$
\psi_{j, k}=2^{-\frac{j}{2}} \psi\left(2^{-j} t-k\right) .
$$

The discrete wavelet transform (DWT) is defined as

$$
c_{j, k}=\int f(t) \psi_{j, k}^{*}(t) d t
$$

where $c_{j, k}$ is called the wavelet coefficient. It may be considered as a time frequency map of the original signal $f(t)$. A multiresolution analysis approach is used in which a discrete scaling function

$$
\phi_{j, k}=2^{-\frac{j}{2}} \phi\left(\frac{t-2^{j} k}{2^{j}}\right)
$$

is defined, together with

$$
d_{j, k}=\int f(t) \phi_{j, k}^{*}(t) d t
$$

where $d_{j, k}$ is called the scaling coefficient which is the sampled version of the original signal. The DWT computes the wavelet coefficients $c_{j, k}$ and $d_{j, k}(j=1, \ldots, J)$ given by

and

$$
c_{j, k}=\sum_{n} x[n] h_{j}\left[n-2^{j} k\right]
$$

$$
d_{j, k}=\sum_{n} x[n] g_{j}\left[n-2^{j} k\right]
$$

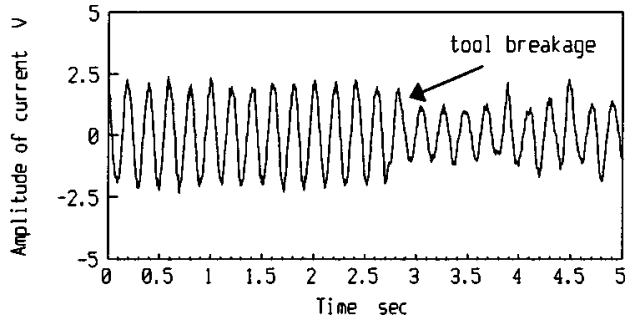

(a)

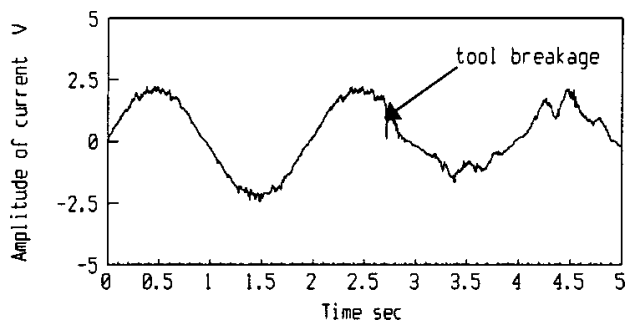

(b)

Fig. 1. Live tool breakage current signals, cutting speed: $250 \mathrm{r} / \mathrm{min}$, feed speed: $30 \mathrm{~mm} / \mathrm{min}$, and drill diameter: $2 \mathrm{~mm}$. (a) Spindle current signal and (b) feed current signal.

where $x[n]$ is a discrete-time series, $h_{j}\left[n-2^{j} k\right]$ is called the discrete wavelets, is equivalent to $2^{-j / 2} \psi\left(2^{-j}\left(t-2^{j} k\right)\right)$. The term $g_{j}\left[n-2^{j} k\right]$ is called the scaling sequence. At the $j$ th resolution, $c_{j, k}$ and $d_{j, k}$ present the approximation and the detail signal $f(t)$. At each resolution $j>0$, the scaling coefficients and the wavelet coefficients are

$$
\begin{aligned}
c_{j+1, k} & =\sum_{n} g[n-2 k] d_{j, k} \\
d_{j+1, k} & =\sum_{n} h[n-2 k] d_{j, k} .
\end{aligned}
$$

\section{B. Tool Breakage Detection by Using Wavelet Transforms}

With reference to Fig. 1(a), when a tool breaks, the amplitude of the spindle motor current drops rapidly. It is a key indicator to reflect the attenuation using some signal processing method, such as time or frequency domain method. But the usual analysis cannot be applied to detect if a tool breaks. In this paper, the Morlet wavelet function is used to deal with the spindle motor current signals, given by

$$
\psi(t)=e^{-\frac{t^{2}}{2}}=e^{i w_{0} t}
$$

Its continuous wavelet is

$$
\psi_{a, b}(t)=\frac{1}{\sqrt{|a|}} e^{-\frac{1}{2}\left(\frac{t-b}{a}\right)^{2}} e^{j w_{0}\left(\frac{t-b}{a}\right)} .
$$

In this equation, the relation between the parameters $a$ and $w_{o}$ is

$$
a=\frac{w_{0} f_{s}}{2 \pi f_{w} f_{0}}
$$

where

$f_{w} \quad$ corresponds to the frequency of the wavelet function;

$f_{s} \quad$ sample frequency of the signals;

$f_{0} \quad$ frequency focused on the energy of the signals. 


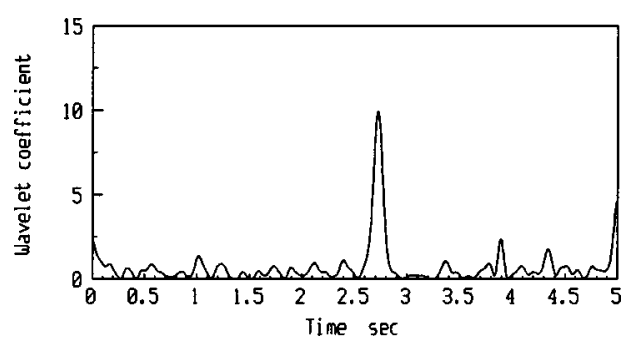

(a)

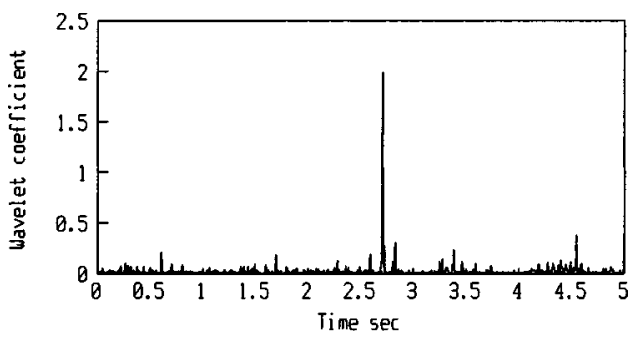

(b)

Fig. 2. WT of current signals, cutting speed: $250 \mathrm{r} / \mathrm{min}$, feed speed: 30 $\mathrm{mm} / \mathrm{min}$, and drill diameter: $2 \mathrm{~mm}$. (a) CWT of the spindle current signal and (b) DWT of feed current, resolution $j=2$.

When a tool is breaking, the feed motor current signals show some spikes [Fig. 1(b)]. At the moment the tool breaks, a big spike appears in the current signals, and then the following signals are not smooth. Based on the features of feed motor current signals, in this paper the DWT is used to analyze the feed motor current signals. The filters used are $h[\cdot]=[0.125,0.375,0.375,0.125]$ and $g[\cdot]=[-2.0,2.0]$.

Above method is used to analyze the current signals of typical tool breakage. Fig. 1(a)-(b) shows the spindle motor current signals and the feed motor current with tool breakage, respectively. Fig. 2(a)-(b) shows the results of the CWT analysis of the spindle motor current and the results of the DWT analysis of the feed motor current at resolution $j=2$, respectively. From the analysis results, the features of tool breakage can be easily extracted using a very simple method. In [17], the tool breakage can be efficiently detected under various kinds of cutting conditions using wavelet transforms.

\section{TOOL WEARS DETECTION USING FUZZY ClASSIFICATION TECHNIQUES}

\section{A. Tool Wear Model}

Based on past studies, tool wear, spindle speed, feed rate, and drill diameter all affect the motor current signals in a cutting process. Moreover, the spindle and feed motor currents can be selected as the features relevant to tool wear states [18]. In the studies, the flank wear is taken as a standard for evaluating the drill wear condition. The tool wear mentioned in the rest of this paper refers to the drill flank wear. In this paper, regression analysis is used to determine the map to spindle current or feed current from spindle speed $v(\mathrm{~m} / \mathrm{min})$, feed rate $f(\mathrm{~mm} / \mathrm{rev})$, and drill diameter $d(\mathrm{~mm})$ when the tool wear $w(\mathrm{~mm})$ is $0.20,0.50$, and 0.80 , respectively. The effect of the cutting variables $v, f$, and $d$ on the current signals, for a sharp tool, is hence assumed to be represented by the following formula [19]:

$$
I=K_{0} v^{a_{1}} f^{a_{2}} d^{a_{3}}
$$

where $I$ is the RMS current, and $K_{0}$ depends on the tool geometry and workpiece material. Taking the logarithmic value of $I$, for the different tool wear values of $0.2,0.5$, and $0.8 \mathrm{~mm}$, we obtain for the spindle motor current $S_{i}$ and feed motor current $F_{i}$ (for $i=1,2,3$ ) in the following relations:

$$
\begin{array}{lr}
S_{1}=a_{01}+a_{11} \ln v+a_{21} \ln f+a_{31} \ln d & \text { wear }=0.2 \mathrm{~mm} \\
S_{2}=a_{02}+a_{12} \ln v+a_{22} \ln f+a_{32} \ln d & \text { wear }=0.5 \mathrm{~mm} \\
S_{3}=a_{03}+a_{13} \ln v+a_{23} \ln f+a_{33} \ln d & \text { wear }=0.8 \mathrm{~mm} \\
F_{1}=b_{01}+b_{11} \ln v+b_{21} \ln f+b_{31} \ln d & \text { wear }=0.2 \mathrm{~mm} \\
F_{2}=b_{02}+b_{12} \ln v+b_{22} \ln f+b_{32} \ln d & \text { wear }=0.5 \mathrm{~mm}
\end{array}
$$

$F_{3}=b_{03}+b_{13} \ln v+b_{23} \ln f+b_{33} \ln d \quad$ wear $=0.8 \mathrm{~mm}$

where subscripts $i=1,2,3$ denote the respective wear values. According to previous experiments, the parameters in (13) and (14), $a_{i j}$ and $b_{i j}(i, j=0,1,2,3)$, can be determined. Thus, knowing the cutting parameters $(v, f, d)$, the values of spindle and feed motor current $\left[S_{i}, F_{i}\right]$ can be estimated for the wear values of $0.2,0.5$, and $0.8 \mathrm{~mm}$ respectively based on the above equations.

\section{B. Fuzzy Classification}

The measured motor currents in real time are defined as the real feature values $\left[S_{0}, F_{0}\right] . S_{0}$ and $F_{0}$ are compared with the estimated features $\left[S_{i}, F_{i}\right]$ for different defined wear states (i.e., $0.2,0.5$, and $0.8 \mathrm{~mm}$ ) in order to evaluate the degree of similarity between the real wear state to any of the estimated wear state. For the spindle current, the following membership $\mu_{S_{i}}$ values are established:

1) if $S_{0}<S_{i}(i=1,2,3)$ then $\mu_{S_{1}}\left(S_{0}\right)=1, \mu_{S_{2}}\left(S_{0}\right)=0$, $\mu_{S_{3}}\left(S_{0}\right)=0$

2) if $S_{1} \leq S_{0} \leq S_{2}$ then $\mu_{S_{1}}\left(S_{0}\right)=\left(S_{2}-S_{0} / S_{2}-S_{1}\right)$, $\mu_{S_{2}}\left(S_{0}\right)=\left(S_{0}-S_{1} / S_{2}-S_{1}\right), \mu_{S_{3}}\left(S_{0}\right)=0$;

3) if $S_{2} \leq S_{0} \leq S_{3}$ the $\mu_{S_{1}}\left(S_{0}\right)=0, \mu_{S_{2}}\left(S_{0}\right)=\left(S_{3}-\right.$ $\left.S_{0} / S_{3}-S_{2}\right), \mu_{S_{3}}\left(S_{0}\right)=\left(S_{0}-S_{2} / S_{3}-S_{1}\right)$

4) if $S_{0}>S_{i}(i=1,2,3)$ then $\mu_{S_{1}}\left(S_{0}\right)=0, \mu_{S_{2}}\left(S_{0}\right)=0$, $\mu_{S_{3}}\left(S_{0}\right)=1$

where $\mu_{S_{i}}\left(S_{0}\right)$ is the membership function of $S_{0}$ associated with the $i$ th grade of tool wear state. The same method may be used to deal with the case for the feed motor current.

The composite result is the average of the summation of the weighted membership functions

$$
\mu_{i}(w)=w_{1} \mu_{S_{i}}\left(S_{0}\right)+w_{2} \mu_{F_{i}}\left(S_{0}\right) \quad(i=1,2,3)
$$

where the weighting coefficient $w_{i}(i=1,2)$ satisfies the normalized condition: $w_{1}+w_{2}=1, w_{1}, w_{2}$ reflect the relative influence of the classification result of the spindle motor current feature and feed motor current feature, respectively. In the present system for the given wear conditions, the coefficients 


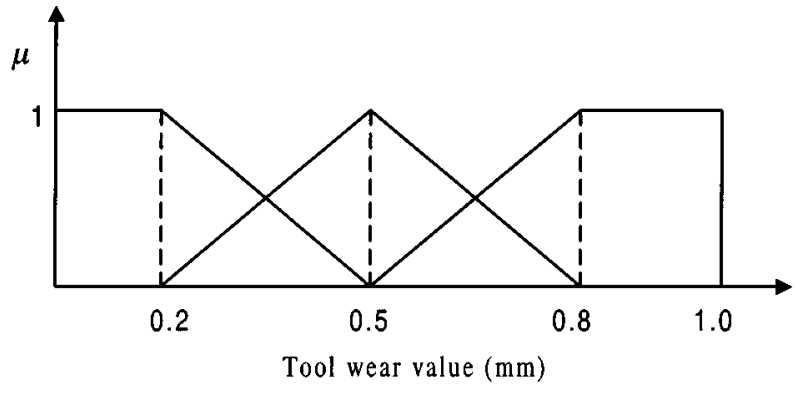

Fig. 3. Fuzzy membership of tool wear states.

are $w_{1}=0.4, w_{2}=0.6$, implying that the feed motor current feature is more important than the spindle motor current feature.

The outputs of the above process are still fuzzy values and they have to be defuzzified. Basically defuzzification is a mapping from a space of fuzzy values into that of the crispy universe. In the present system, the centroid defuzzification method is selected, which produces the center of area of the possibility distribution of the inferenced output. Shown in Fig. 3 is the membership function of the tool wear states. Therefore, the estimated tool wear states can be obtained by using the following center-of-gravity (centroid) defuzzification rule:

$$
\text { wear }=\frac{\int_{w} \mu(w) w d w}{\int_{w} \mu(w) d w}
$$

where wear represents the numerical value of tool wear states.

To sum up, a flowchart of the tool wear classification is shown in Fig. 4.

\section{EXPERIMENTAL RESULTS}

\section{A. Experimental Setup}

The schematic diagram of the experimental setup is shown in Fig. 5. The monitoring system integrates two modules including tool breakage detection and tool wear detection, and is fixed on a Machine Center Makino-FNC74-A20. The four axes (spindle, $\mathrm{X}, \mathrm{Y}$, and $\mathrm{Z}$ ) of the machine with recalculating ball screw drives are directly driven by permanent-magnet synchronous ac servomotors. The ac servo motor current signals of the Machine Center were measured through a Hall-effect-current sensor. The signals were initially passed through low-pass filters (with a cutoff frequency of $500 \mathrm{~Hz}$ ), and then sent to a personal computer via an $\mathrm{A} / \mathrm{D}$ converter.

A successful tool breakage and wear detecting method must be sensitive to the change of tool conditions, and insensitive to the variations of cutting conditions. Hence, cutting tests were conducted at different cutting conditions to evaluate the performance of the proposed method. Table I shows the experimental cutting conditions.

\section{B. Results and Discussions}

In this monitoring system, the tool breakage detection and tool wear detection are very different. Tool breakage detection is based on the wavelet transform method whereas tool wear detection is based on the fuzzy classification method. They both detect the tool conditions by measuring the spindle and feed motor currents. Taking the difference of schemes into account,

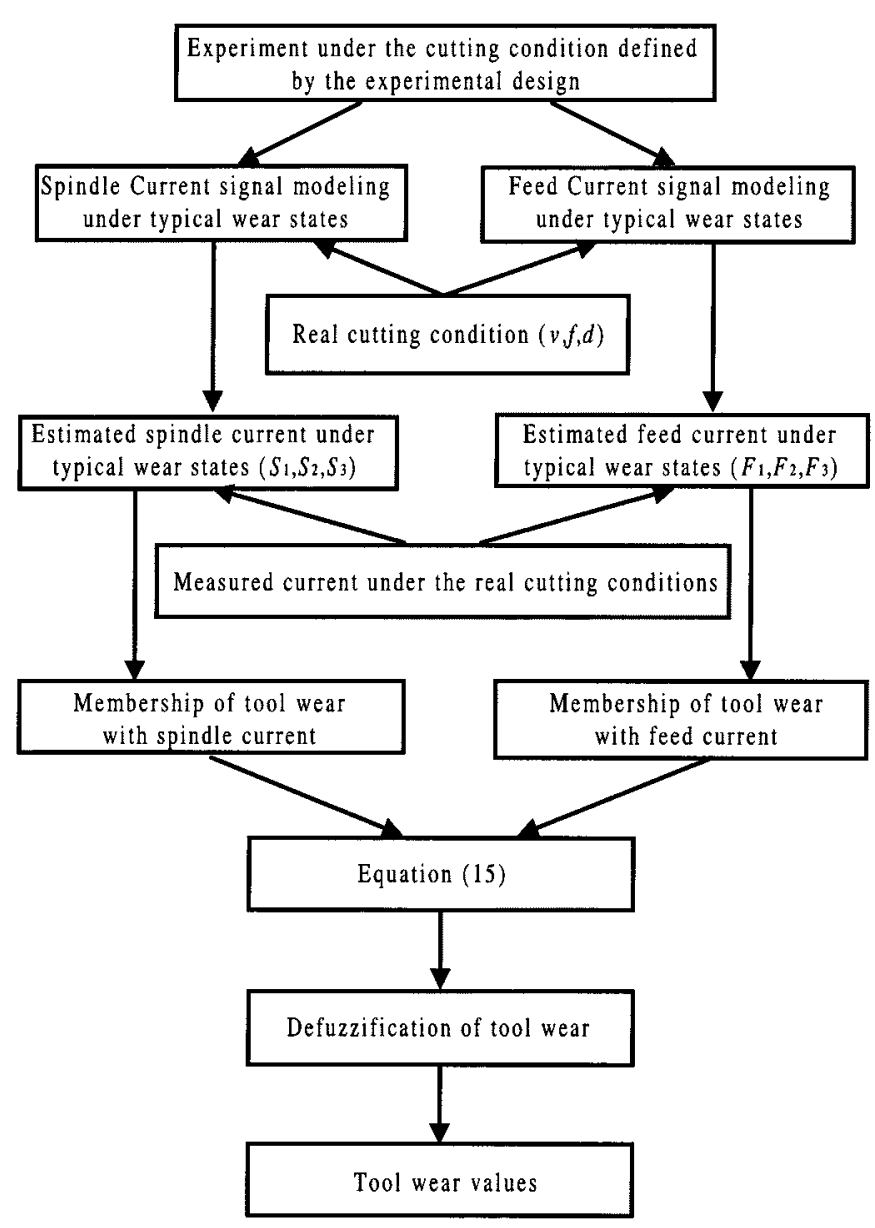

Fig. 4. Flowchart of the tool wear estimation.

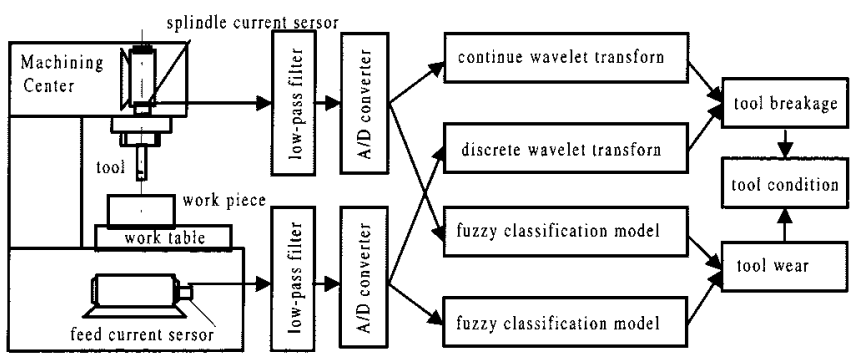

Fig. 5. Schematic diagram of the experimental setup.

TABLE I

EXPERIMENTAL CONDITION

\begin{tabular}{ll}
\hline TOOL & Material: high-speed steel \\
\hline CUTTING CONDITIONS & Spindle speed: $250,300,450(\mathrm{rpm})$ \\
& Feed speed: $\quad 25,30,45(\mathrm{~mm} / \mathrm{min})$ \\
& Drill diameters: $2,3,4.5,7.2,9.6 \mathrm{~mm}$ \\
& Flank wear: $\quad 0.2,0.5,0.8(\mathrm{~mm})$ \\
& Without coolant \\
\hline WORKPIECE & 45\# steel \\
\hline
\end{tabular}

the tool breakage detection and the tool wear detection are separately tested. 
TABLE II

CUTTING CONDITION AND EXPERIMENTAL RESULTS

\begin{tabular}{c|c|c|c|c}
\hline $\begin{array}{c}\text { DRILL DIAMETER } \\
(\mathrm{mm})\end{array}$ & $\begin{array}{c}\text { ROTATIONAL } \\
\text { SPEED (rpm) }\end{array}$ & $\begin{array}{c}\text { FEED SPEED } \\
(\mathrm{mm} / \mathrm{min})\end{array}$ & $\begin{array}{c}\text { FACTUAL TOOL } \\
\text { BREAKAGE TIMES }\end{array}$ & $\begin{array}{c}\text { TOOL MONITORING } \\
\text { SYSTEM REPORT TIMES }\end{array}$ \\
\hline 2.0 & 300 & 30 & 50 & 50 \\
3.0 & 450 & 45 & 100 & 98 \\
4.5 & 300 & 30 & 50 & 50 \\
7.2 & 450 & 25 & 50 & 50 \\
9.6 & 250 & 25 & 20 & 20 \\
\hline
\end{tabular}

First, the monitoring system is used to detect tool breakage. To detect the tool breakage efficiently in a drilling process, the monitoring system should be fit into the kinds of cutting conditions. Cutting tests were conducted under various cutting conditions. The test results are shown in Table II. From the test results, the successful detection rates all are over $95 \%$ under different cutting conditions. For small-diameter drills, such as 2 and 3 $\mathrm{mm}$, the successful detection rates are also very good. From past studies, the motor current signals are only fit to detect some big diameter drills (with diameter over $5 \mathrm{~mm}$ ). Thus, the extracted features derived from the wavelet transform of the motor current are useful. They can be effectively used to detect tool breakage. In addition, these results also showed that the features extracted were not too sensitive to the change of cutting conditions. In brief, the experimental results show that the tool breakage detection module of the monitoring system can meet the need of the drilling applications.

For tool wear detection, a total of 70 cutting tests were collected under various cutting conditions. Fifty samples were randomly picked as the learning samples for calculating the parameters of (13) and (14), $A=\left[a_{i j}\right]$ and $B=\left[b_{i j}\right]$. Twenty samples were used as the test samples in the classification phase. According to the experimental results, the parameters $A$ and $B$ can be calculated by using the least-squares method as follows:

$$
\begin{aligned}
& A=\left[\begin{array}{llll}
2.6405 & 0.1502 & 0.0931 & 0.1807 \\
2.6884 & 0.1679 & 0.1185 & 0.3111 \\
2.7658 & 0.1826 & 0.1536 & 0.3549
\end{array}\right] \text { and } \\
& B=\left[\begin{array}{llll}
2.7058 & 0.0953 & 0.0659 & 0.2084 \\
2.7418 & 0.1828 & 0.1156 & 0.3136 \\
2.8641 & 0.1933 & 0.1692 & 0.3435
\end{array}\right]
\end{aligned}
$$

The correlation coefficient was always over 0.97 , close to unity. It is indicated that the relationship between the current signals and the cutting parameters is well represented by the proposed models. Twenty additional tests were conducted to examine the feasibility of using the above models to estimate the tool wear states. In order to make clear the reliability of the above method, the comparison of actual tool wear values with estimated tool wear values is shown in Fig. 6. In the test samples, the error rates are up to $15 \%$. The success rate of tool wear monitoring is thus as high as $85 \%$. The results are acceptable and the proposed method can be applied in practical cutting processing, this reason is that the relative error of actual measurement of tool wear is close to $90 \%$.

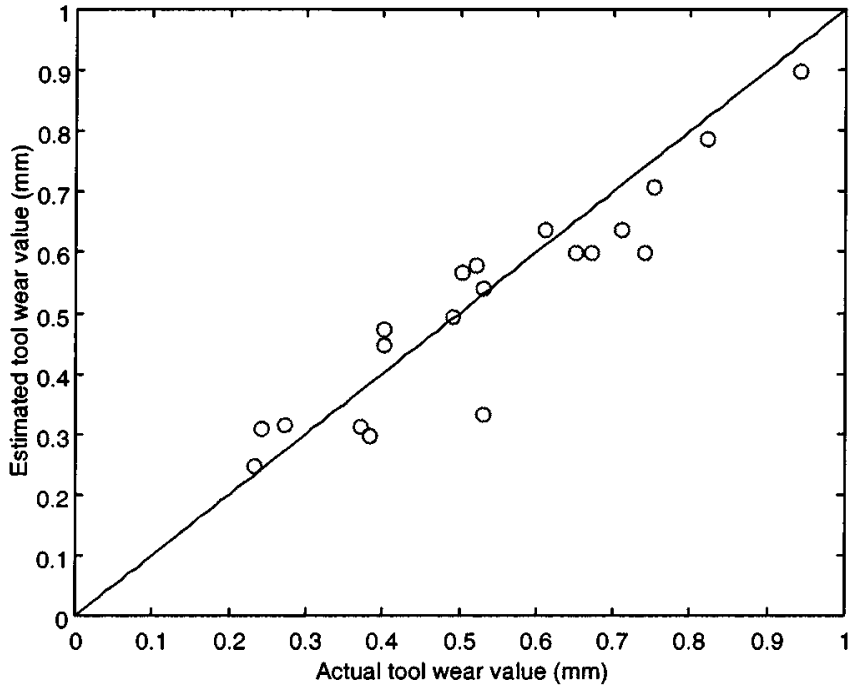

Fig. 6. Comparison of actual tool wear values with estimated tool wear values.

\section{CONCLUSION}

An intelligent tool condition monitoring system is developed based on ac servo motor current signals measured using simple current sensors. The monitoring system possesses the following advantages:

1) monitoring method is very simple and effective;

2) cost of the system is very low;

3) monitoring system does not interfere with cutting processing.

From the experimental results, the following conclusions can be made.

1) The continuous and discrete wavelet transforms are useful to decompose the spindle and feed motor current signals, respectively. The features of tool breakage can be easily extracted from the decomposed signals so as to effectively detect the tool breakage in drilling.

2) The tool wear models of the relationships between the current signals and the cutting parameters under different tool wear states (i.e., wear $0.2,0.5,0.8 \mathrm{~mm}$ ) are established based on partial experimental design and regression analysis. The fuzzy classification method is useful to calculate the membership degree of tool wear by using the measured motor currents. Finally, the tool wear value can be estimated based on the above method.

3) The monitoring system includes tool breakage detection and tool wear detection. The experimental results show that the success rate of tool breakage detection is over $95 \%$, and the error of tool wear detection is below $15 \%$. These results strongly show that the monitoring system may be employed in practical drilling applications.

\section{ACKNOWLEDGMENT}

The authors would like to express their appreciation for the useful suggestions put forward by the anomymous referees and an Associate Editor. 


\section{REFERENCES}

[1] D. Li and J. Mathew, "Tool wear and failure monitoring techniques for turning-A review," Int. J. Mach. Tools Manufact., vol. 30, no. 4, pp. 579-598, 1990.

[2] X. Li and Z. Yuan, "Tool wear monitoring with wavelet packet transform-fuzzy clustering method," Wear, vol. 219 , no. 2, pp. 145-154, 1998.

[3] M. A. Mannan, S. Broms, and B. Lindustrom, "Monitoring and adaptive control of cutting process by means of motor power and current measurements," Ann. CIRP, vol. 38, no. 1, pp. 347-350, 1989.

[4] M. A. Mannan and N. Tomas, "The behavior of static torque and thrust due to tool wear in drilling," Tech. Rep., North Amer. Manufact. Res. Inst. SME, 1997.

[5] G. Byrne, D. Dornfeld, I. Inasaki, G. Ketteler, W. Konig, and R. Teti, "Tool condition monitoring (TCM) - The status of research and industrial application," Ann. CIRP, vol. 44, no. 2, pp. 541-567, 1995.

[6] X. Li, Y. Yao, and Z. Yuan, "On-line tool condition monitoring system with wavelet fuzzy neural network," J. Intell. Manufact., vol. 8, pp. 271-276, Aug. 1997.

[7] W. Gong, T. Obikawa, and T. Shirakashi, "Monitoring of tool wear states in turning based on wavelet analysis," JSME Int. J. Ser. III-Vibr. Contr. Eng. Ind., vol. 40, pp. 447-453, Sept. 1997.

[8] G. S. Hong, M. Rahman, and Q. Zhou, "Using neural network for tool condition monitoring based on wavelet decomposition," Int. J. Mach. Tools Manufact., vol. 36, pp. 551-566, May 1996.

[9] Ya Wu and R. Du, "Feature extraction and assessment using wavelet packets for monitoring of machining processes," Mech. Syst. Signal Process., vol. 10, pp. 29-53, Jan. 1996.

[10] Q. Zhou, G. S. Hong, and M. Rahman, "New tool life criterion for tool condition monitoring using a neural network," Eng. Applicat. Artif. Intell., vol. 8, pp. 579-588, Oct. 1995.

[11] N. Kasashima, K. Mori, G. H. Ruiz, and N. Taniguchi, "Online failure detection in face milling using discrete wavelet transform," Ann. CIRP, vol. 44, no. 1 , pp. 483-487, 1995.

[12] I. N. Tansel, C. Mekdeci, and C. McLaughlin, "Detection of tool failure in end milling with wavelet transformations and neural networks (WTNN)," Int. J. Mach. Tools Manufact., vol. 35, pp. 1137-1147, Aug. 1995.

[13] I. N. Tanasel, C. Mekdeci, C. Rodriguez, and O. Uragun, "Monitoring drill conditions with wavelet based encoding and neural networks," Int J. Mach. Tools Manufact., vol. 33, pp. 559-575, Aug. 1993.

[14] Y. Koren, T. Ko, and A. Galip, "Flank wear estimation under varying cutting conditions," J. Dyn. Syst., Meas., Contr., vol. 113, pp. 300-307, 1991.

[15] G. Strang and T. Nguyen, Wavelets and Filter Banks. Cambridge, U.K.: Wellesley-Cambridge Press, 1996.

[16] Y. Meyer, Wavelets: Algorthms and Applications. Phiadelphia, PA SIAM, 1993.

[17] X. Li, "On-line detection of the breakage of small diameter drills using current signature wavelet transform," Int. J. Mach. Tools Manufact., vol. 39, no. 9 , pp. $157-164,1999$.
[18] X. Li and S. K. Tso, "Drill wear monitoring based on current signals," Wear, vol. 231, pp. 172-178, 1999.

[19] X. Li, "Identification of tool wear states with fuzzy classification," Int. J. Comput. Integr. Manufact., vol. 12, no. 6, pp. 503-509, 1999.

Xiaoli Li was born in Jiangxi, China, in 1970. He received the B.E. and M.E degrees in mechanical engineering from Kunming University of Science and Technology, Kunming, China, in 1992 and 1995, respectively, and the Ph.D. degree in mechanical engineering from Harbin Institute of Technology, Harbin, China, in 1998.

He is currently a Research Fellow with the Department of Manufacturing Engineering and Engineering Management, City University of Hong Kong, Hong Kong. His main areas of research are manufacturing process monitoring, machining error compensation, fuzzy systems, neural networks, and wavelet transform and its applications.

Shiu Kit Tso (SM'81) joined the City University of Hong Kong as Professor of mechatronics and automation in 1995 after a period of long service with the University of Hong Kong. He has carried out research in the broad field of industrial electronics and automation and control and supervised numerous Ph.D. M.Phil., and M.Sc. students. He has published more than 250 refereed scientific papers. He has worked in the industrial electronics and control industry and served as consultant for a number of government and industrial projects. $\mathrm{He}$ has held visiting professorships in many universities including the University of Toronto, University of Waterloo, and National University of Singapore. He is currently Director of the Centre for Intelligent Design, Automation, and Manufacturing, City University of Hong Kong.

Dr. Tso is a Fellow of IEE and HKIE.

Jun Wang (S'89-M'90-SM'93) received the B.S. degree in electrical engineering and the M.S. degree in systems engineering from Dalian University of Technology, China, and the Ph.D. degree in systems engineering from Case Western Reserve University, Cleveland, OH.

$\mathrm{He}$ is an Associate Professor and the Director of Computational Intelligence Laboratory, Department of Automation and Computer-Aided Engineering, Chinese University of Hong Kong. Prior to this, he was an Associate Professor with the University of North Dakota, Grand Forks. His current research interests include neural networks and their engineering applications.

Dr. Wang is an Associate Editor of the IEEE Transactions on NEURAL NETWORKS 